\title{
Role of P57KIP2 Immunohistochemical Expression in Histological Diagnosis of Hydatidiform Moles
}

\author{
Surang Triratanachat ${ }^{1}$, Pattawan Nakaporntham ${ }^{2}$, Patou Tantbirojn ${ }^{2}$, Shanop \\ Shuangshoti ${ }^{3}$, Ruangsak Lertkhachonsuk ${ }^{2 *}$
}

\begin{abstract}
Purpose: To determine the significance of P57KIP2 immunohistochemistry expression in the histopathological diagnosis of hydatidiform mole. Materials and Methods: Hydatidiform mole patients at King Chulalongkorn Memorial Hospital between January 1999 and December 2011 were recruited. Two gynecologic pathologists reviewed histopathologic slides to confirm diagnosis. Formalin-fixed, paraffin-embedded tissue sections were stained using a bstandard immunostaining system with monoclonal antibodies against P57KIP2 protein. Correlations among pathological features, immunohistochemical expression and clinical data were analyzed. Results: One hundred and twenty-seven hydatidiform mole patients were enrolled. After consensus review, 97 cases were diagnosed as complet (CHM) and 30 cases as partial (PHM). Discordance between the first and final H\&E diagnoses was found in 19 cases $(14.9 \%, \mathrm{k}=0.578)$. Significant pathological features to classify the type of hydatidiform mole are central cisterns, trophoblastic proliferation, trophoblastic atypia, two populations of villi, fetal vessels and scalloped borders. After performing immunohistochemistry for P57 KIP2, 107 cases were P57KIP2 negative and 20 cases positive. Discordant diagnoses between final H\&E diagnosis and P57KIP2 immunohistochemistry was identified in 12 cases $(9.4 \%)$. Sensitivity of final $\mathrm{H} \& \mathrm{E}$ diagnosis for CHM was 89.7\% ; specificity was $95.0 \%$. PHM sensitivity and specificity of final $\mathrm{H} \& \mathrm{E}$ diagnosis was $\mathbf{9 5 . 0 \%}$ and $89.7 \%$, respectively. Conclusions: Histopathological diagnosis alone has certain limitations in accurately defining types of hydatidiform mole; P57KIP2 immunohistochemistry is practical and can be a useful adjunct to histopathology to distinguish CHM from non-CHM.
\end{abstract}

Asian Pac J Cancer Prev, 17 (4), 2061-2066

\section{Introduction}

The hydatidiform mole is a term used for a group of pregnancy-related tumors. The incidence varies greatly among different parts of the world with an approximate risk of 1 per 1000 pregnancies (Ngan et al., 2012). The reported incidence of hydatidiform mole is high in Asia. At King Chulalongkorn Memorial Hospital $(\mathrm{KCMH})$, Bangkok, Thailand, the reported incidence is approximately 1 in 600 pregnancies (Wairachpanich et al., 2015). Majority of patients with hydatidiform mole have spontaneous regression after evacuation. However, in some patients, serum hCG levels rise or plateau after treatment and are considered postmolar gestational trophoblastic neoplastic neoplasia (GTN).

There are two subtypes of hydatidiform moles, complete hydatidiform mole (CHM) and partial hydatidiform mole (PHM). Incidence of CHM and PHM are different. Several reports show that PHM incidence is around three times higher than CHM (Jeffers et al., 1993; Seckl et al., 2013). The study from our institute reveals that incidence of CHM is nearly seven times higher than PHM which differ from other previous reports (Lertkhachonsuk et al., 2012b). Moreover, incidence of postmolar GTN after CHM and PHM in our institute are $29.7 \%$ and $23.3 \%$, respectively while other reports show postmolar GTN 8-29\% after CHM and 1-5 \% after PHM (Hancock et al., 2006; Seckl et al., 2010).

Sub-classification of hydatidiform mole as CHM and PHM are important for determining the risk of GTN and clinical management. Diagnosis of hydatidiform mole is based on morphology (hematoxylin and eosin staining) which has high inter-observer and intra-observer variability. Moreover, diagnosis usually depends on the experience of pathologists (Javey et al., 1979; Howat et al., 1993; Fukunaga et al., 2005). Furthermore, current hydatidiform mole evacuation is carried out in early gestational age, especially before well-established classic morphologic features. These reasons have led to more difficulties in diagnosis and classification of hydatidiform mole.

Varieties of ancillary techniques have been used to

${ }^{1}$ Division of Gynecologic Pathology and Cytology, ${ }^{2}$ Placenta Related Disease Research Unit, Department of Obstetrics and Gynecology, ${ }^{3}$ Department of Pathology, Faculty of Medicine, Chulalongkorn University, Bangkok, Thailand *For correspondence: drruang9@yahoo.com,ruangsak@chula.md 
improve the diagnosis of hydatidiform mole, including DNA flow cytometry, karyotype, fluorescent in situ hybridization, immunohistochemistry for P57KIP2 gene, and PCR amplification of short tandem repeat loci. P57KIP2 (CDKN1C, Kip2) is a protein in the nucleus which is encoded by CDKN1C gene (tumor suppressor gene) on chromosome 11 . The protein P57KIP2 is strongly paternally imprinted and expressed only from the maternal allele (maternally expressed gene). CHM is absent in P57KIP2 protein because it contains only paternal genes, while PHM presents P57KIP2 protein because it contains both the maternal and paternal genomes. Immunohistochemistry of P57KIP2 can be used in differential diagnoses of CHM from non-CHM (Merchant et al., 2005; Garcia-Barriola et al., 2008; Hoffner et al., 2008; Pateras et al., 2009; Sasaki et al., 2015). Furthermore, the P57KIP2 immunohistochemistry is not complicated and widely available in clinical practice.

This study was aimed to determine the utility of P57KIP2 immunohistochemistry in hydatidiform mole and to investigate pathological features that can differentiate between complete and partial hydatidiform moles.

\section{Materials and Methods}

\section{Patients and Samples}

After gaining approval from Institutional Review Board of Research Affairs, Faculty of Medicine, Chulalongkorn University No.563/54, patients treated for hydatidiform mole at King Chulalongkorn Memorial Hospital from January 1, 1999 to December 31, 2011 were reviewed. This study recruited available paraffin embedded specimens from hydatidiform mole patients. Exclusion criteria was comprised of previous treatment GTN and unavailable paraffin embedded specimens or no available tissue to evaluate immunohistochemistry. Two gynecologic pathologists independently reviewed hematoxylin and eosin (H\&E) slides based on standard pathological criteria. Eight features of pathological morphology were evaluated, classified as positive if there was diffuse expression, and recorded in each case. Discordant diagnoses and re-screened was carried out by two pathologists. Consensus diagnosis was based on H\&E stained slides.

Paraffin-embedded specimens were collected and prepared for immunohistochemistry stain using a mouse monoclonal antibody against the P57KIP2Kip2 protein [Ab-3 (clone KP39), Neomarker, Fremont, CA, USA] at 1:200 dilution. Two micron thick sections underwent overnight baking at $60^{\circ} \mathrm{c}$. Deparaffinization and heatpretreatment antigen retrieval was carried out with Envision TM FLEX target retrieval solution high $\mathrm{pH}$ with
PT Link (Dako, Denmark). All sections were stained using an autostainer, Link 48 (DAKO, Denmark) and detected with Envision TM FLEX/HRP (DAKO, Denmark). Diaminobenzidine (DAB) was used as chromogen. Negative control was performed by substituting the primary antibody with non-immune sera. Decidua and extra-villous trophoblast served as internal positive control. Appropriate positive and negative controls were run simultaneously. The quality of immunohistochemistry was evaluated and interpreted independently by two gynecologic pathologists. Both were blinded from histological diagnoses. The P57KIP2 expression was evaluated in the nucleus of villous stromal cells and cytotrophoblasts. Positive results were noted when more than $10 \%$ staining of these cells were present.

Clinical characteristics and outcomes of patients were correlated with histologic and immunohistochemistry results. Qualitative variables are shown as percentages. Quantitative variables are displayed with median arithmetic and SD. Statistical significance for qualitative variables was analyzed with Pearson's correction using CI of $95 \%(\mathrm{p}<0.05)$. Statistical significance for quantitative variables was analyzed with Fisher's exact test using CI of $95 \%(\mathrm{p}<0.05)$. Statistical analysis was calculated with SPSS version 17.0 (Chicago IL, USA)

\section{Results}

There were 139 cases of hydatidiform mole from January 1999 to December 2011. Twelve cases were excluded from this study due to loss of paraffin blocks ( 9 cases) and inadequate molar tissue (3 cases) in paraffin blocks. One hundred and twenty-seven patients with hydatidiform mole were enrolled in the study.

\section{Histologic findings}

A total of 127 cases were classified at the first diagnosis; 103 cases of CHM and 24 cases of PHM. H\&E slides from all cases were reviewed by two gynecologic pathologists. Discordant diagnosis in hydatidiform mole type between the two pathologists was 32 cases $(25.1 \%$, $\mathrm{k}=0.373$ ). Final pathological diagnosis was reached by consensus of the two pathologists. Discordant diagnoses between the two pathologists were re-classified as 15 cases of CHM (15.5\% of CHM) and 17cases of PHM (56.7\% of PHM). From 127 cases, 97 were re-classified as CHM and 30 cases PHM. Discordant diagnosis between the first and final diagnoses by $\mathrm{H} \& \mathrm{E}$ was found in 19 cases $(14.9 \%$, $\mathrm{k}=0.578$ ). Classified subtypes of hydatidiform mole by pathological diagnosis are summarized in Table 1.

Eight features of pathological morphology were evaluated (Figure1). Central cistern, trophoblastic

Table 1. Classification Subtype of Hydatidiform Mole by Histological Diagnosis and p57 Immunohistochemistry $(\mathbf{n}=127)$

\begin{tabular}{cccccc}
\hline & \multicolumn{4}{c}{ Diagnosis by H\&E } \\
\cline { 2 - 5 } & First & Revision by two pathologists & Final & Final diagnosis by p57 \\
\cline { 2 - 5 } & Histologic diagnosis & Pathologist 1 & Pathologist 2 & Histologic diagnosis \\
\hline CHM & 103 & 88 & 102 & 97 & 30 \\
PHM & 24 & 39 & 25 & 107 & 20 \\
\hline
\end{tabular}


Table 2. Pathological Morphology and Classification Subtype of Hydatidiform Mole (n = 127)

\begin{tabular}{|c|c|c|c|c|}
\hline \multirow{3}{*}{ Pathological morphology } & \multicolumn{3}{|c|}{ Final diagnosis by p57 } & \multirow{3}{*}{ p-value } \\
\hline & Total & $\mathrm{CHM}$ & PHM & \\
\hline & $(n=127)$ & $(n=107)$ & $(n=20)$ & \\
\hline Hydropic villi & $120(94.5 \%)$ & $102(95.3 \%)$ & $18(90.0 \%)$ & 0.39 \\
\hline Central cistern & $110(86.6 \%)$ & $99(92.5 \%)$ & $11(55.0 \%)$ & $<0.01$ \\
\hline Trophoblastic proliferation & $112(88.2 \%)$ & $103(96.3 \%)$ & $9(45.0 \%)$ & $<0.01$ \\
\hline Atypia of IT & $106(83.5 \%)$ & $99(92.5 \%)$ & $7(35.0 \%)$ & $<0.01$ \\
\hline Presence of fetal tissue & $55(43.3 \%)$ & $37(34.6 \%)$ & $18(90.0 \%)$ & $<0.01$ \\
\hline Two populations of villi & $44(34.6 \%)$ & $29(27.1 \%)$ & $15(75.0 \%)$ & $<0.01$ \\
\hline Scalloped border & $62(48.8 \%)$ & $47(43.9 \%)$ & $15(75.0 \%)$ & 0.01 \\
\hline Trophoblastic inclusion & $84(66.1 \%)$ & $68(63.6 \%)$ & $16(80.0 \%)$ & 0.15 \\
\hline
\end{tabular}

Table 3. Patient Characteristics $(n=127)$

\begin{tabular}{|c|c|c|c|c|}
\hline \multirow{3}{*}{ Characteristics } & \multicolumn{3}{|c|}{ Number of patients (\%) } & \multirow{3}{*}{ p-value } \\
\hline & Total & Negative p57* & Positive p57** & \\
\hline & $(n=127)$ & $(\mathrm{n}=107)$ & $(n=20)$ & \\
\hline \multicolumn{5}{|l|}{ Age } \\
\hline Mea \pm SD & $29.3 \pm 9.4$ & $28.7 \pm 9.8$ & $33.4 \pm 5.8$ & \multirow[t]{4}{*}{0.01} \\
\hline$<20$ years & $19(15.0 \%)$ & $19(17.8 \%)$ & - & \\
\hline $20-39$ years & $84(66.1 \%)$ & $69(64.5 \%)$ & $15(75.0 \%)$ & \\
\hline$\geq 40$ years & $24(18.9 \%)$ & $19(17.8 \%)$ & $5(25.0 \%)$ & \\
\hline \multicolumn{5}{|l|}{ Parity } \\
\hline Nulliparity & $41(32.8 \%)$ & $35(32.7 \%)$ & $6(33.3 \%)$ & \multirow[t]{2}{*}{$>0.05$} \\
\hline Multiparity & $84(67.2 \%)$ & $72(67.3 \%)$ & $12(66.7 \%)$ & \\
\hline \multicolumn{5}{|l|}{ GA at diagnosis } \\
\hline Mean $\pm \mathrm{SD}$ & $13.1 \pm 5.1$ & $12.6 \pm 5.0$ & $15.6 \pm 4.6$ & \multirow[t]{3}{*}{$>0.05$} \\
\hline$\leq 12$ weeks & $65(39.2 \%)$ & $61(57.0 \%)$ & $4(20.0 \%)$ & \\
\hline$>12$ weeks & $58(60.8 \%)$ & $45(42.1 \%)$ & $13(65.0 \%)$ & \\
\hline \multicolumn{5}{|c|}{ Pretreatment hCG $(\mathrm{mIU} / \mathrm{ml})$} \\
\hline Median & 198,660 & 210,000 & 49,370 & \multirow[t]{3}{*}{$>0.05$} \\
\hline$\leq 105$ & $35(29.9 \%)$ & $26(24.8 \%)$ & $9(75.0 \%)$ & \\
\hline$>105$ & $82(70.1 \%)$ & $79(75.2 \%)$ & $3(25.0 \%)$ & \\
\hline \multicolumn{5}{|l|}{ Treatment } \\
\hline Suction curettage & $113(89 \%)$ & $94(87.9 \%)$ & $19(95 \%)$ & \multirow{8}{*}{0.01} \\
\hline $\mathrm{TAH}$ & $14(11 \%)$ & $13(12.1)$ & $1(5.0 \%)$ & \\
\hline Postmolar GTN & $35(27.6 \%)$ & $34(31.8 \%)$ & $1(5.0 \%)$ & \\
\hline \multicolumn{4}{|c|}{ Treatment postmolar GTN } & \\
\hline None & $1(2.9 \%)$ & $1(2.9 \%)$ & - & \\
\hline Single CMT & $24(68.6 \%)$ & $24(70.6 \%)$ & - & \\
\hline Combination CMT & $7(20.0 \%)$ & $6(17.6 \%)$ & $1(100.0 \%)$ & \\
\hline Surgery & $3(8.6 \%)$ & $3(8.8 \%)$ & - & \\
\hline
\end{tabular}

*Negative p57 immunohistochemistry = CHM; ** Positive p57 immunohistochemistry = Non-CHM

proliferation, atypia of intermediate trophoblast, presence of fetal vessels, two populations of villi $(\mathrm{p}<0.01)$ and scalloped border $(\mathrm{p}=0.01)$ are significant features for differential diagnosis types of hydatidiform mole. Hydropic villi $(\mathrm{p}=0.39)$ and trophoblastic inclusion $(p=0.15)$ are insignificant features for differentiating CHM from PHM. Hydropic villi, central cistern, trophoblastic proliferation and atypia of trophoblast are presented in CHM more often than in PHM. Whereas, fetal tissue, two populations of villi, scalloped border and trophoblastic inclusion are presented more in PHM than CHM. Pathological morphology and classification of hydatidiform mole are summarized in Table 2.

\section{Immunohistochemical Findings}

After histological revision, 127 cases of hydatidiform mole were eventually reclassified as 97 cases of CHM, and 30 cases of PHM. Following immunohistochemistry (IHC) staining for P57KIP2 (Figure2), discordant diagnosis between histopathology and IHC was identified in 12 cases (9.4\%); 1 case of CHM exhibits positive P57KIP2 and 11 cases of PHM display negative P57KIP2. Sensitivity of final diagnosis by H\&E for CHM and PHM were $89.7 \%$ and $95.0 \%$, respectively. Specificity of the final diagnosis by H\&E for CHM and PHM were $95.0 \%$ and $89.7 \%$, respectively.

Patient characteristics and outcomes are summarized in Table 3. The mean age was 29.3 years. Almost all patients were on first or second pregnancies. The mean gestational age at diagnosis was 13.1 weeks. The most common clinical presentation was abnormal vaginal bleeding. Median Beta-hCG level was 198,660 mIU/ml. 


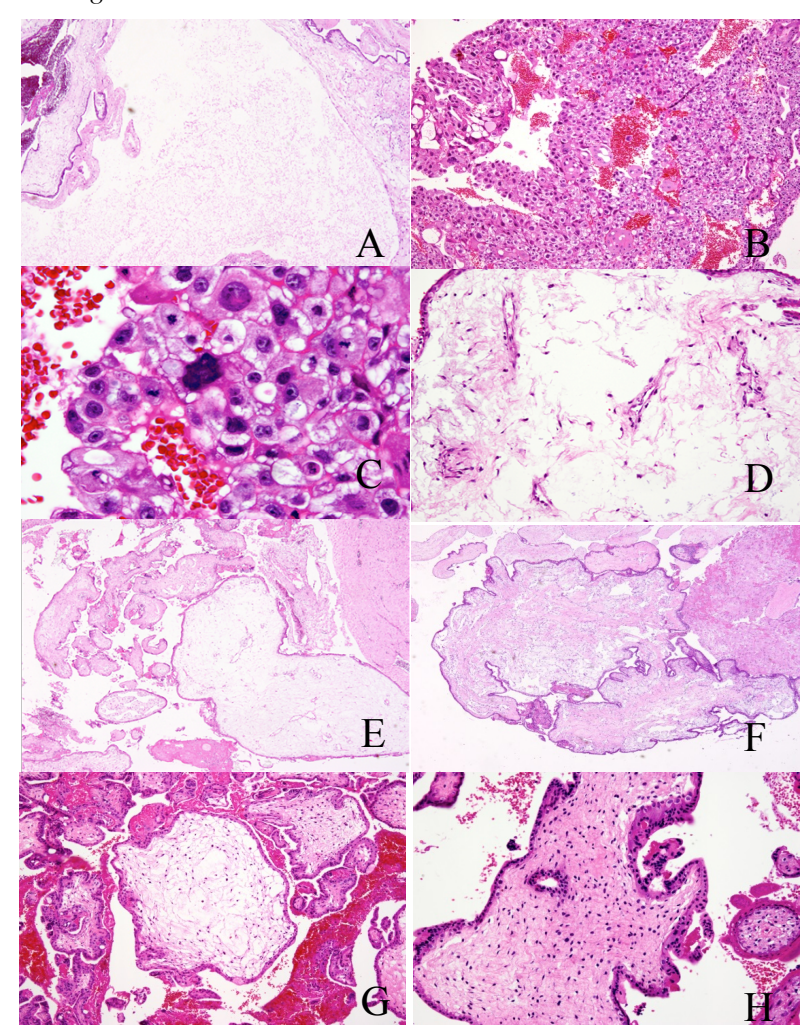

Figure 1. Histologic features of hydatidiform mole. A) central cisterns; B) trophoblastic proliferation; C) trophoblastic atypia; D) presence of fetal vessels; E) two-population of villi; F:scalloped border; $\mathrm{G}$ ) hydropic villi; $\mathrm{H}$ ) trophoblastic inclusion

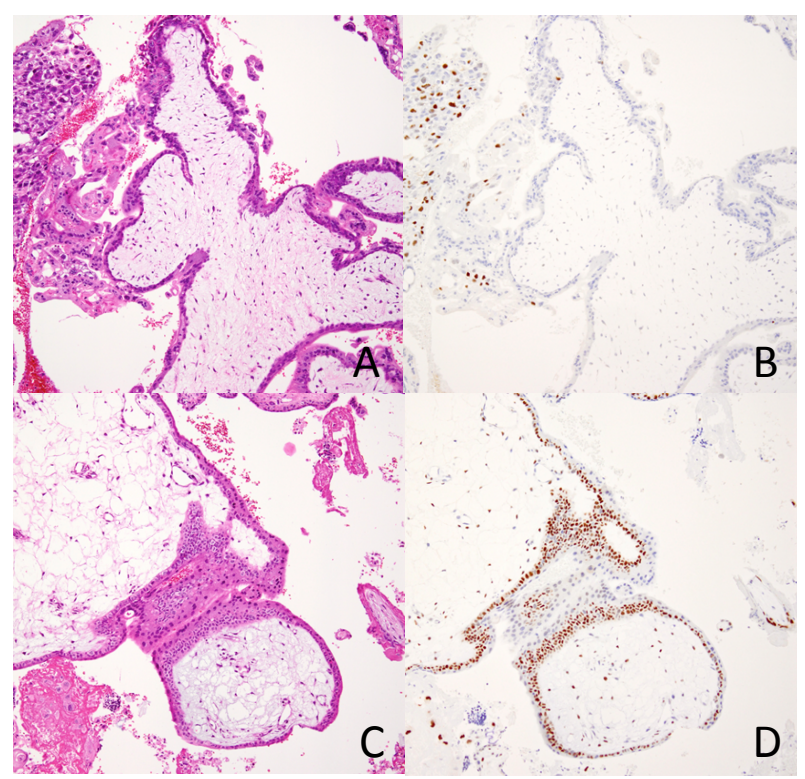

Figure 2. H\&E Staining, p57 Immunohistochemistry of Complete Hydatidiform Mole. (A and B) and H\&E staining, p57 immunohistochemistry of partial hydatidiform mole (C and D)

Patients with CHM were significantly younger than those with PHM (28.7 vs. 33.4 years, $\mathrm{p}=0.01)$. The average gestational age at diagnosis and median pretreatment hCG showed no significant difference between $\mathrm{CHM}$ and PHM. Primary treatment was suction and curettage in most patients. This study found 35 cases of postmolar GTN (28.9\%). According to the final diagnosis by $\mathrm{H} \& \mathrm{E}$, there were 31 cases (32.0\%) of postmolar GTN after CHM and 4 cases $(13.3 \%)$ after PHM. However, postmolar GTN after negative P57KIP2 IHC hydatidiform mole was identified in 34 cases from 107 cases $(31.8 \%)$ and after positive P57KIP2 IHC hydatidiform mole was 1 out of 20 cases $(5.0 \%)$. From thirty-four cases of postmolar GTN, 24 cases (70.6\%) were treated with single agent chemotherapy, 7 cases (20.6\%) with combination chemotherapy and in 3 cases $(8.8 \%)$, surgery was conducted. All patients had successful remission after treatment.

\section{Discussion}

Sub-classification of hydatidiform mole was associated with the risk of postmolar GTN and clinical management. Differentiated type of hydatidiform mole is usually based on histopathology. The classic histologic features of CHM are villous hydrops (extensive cavitation), trophoblastic proliferation (circumferential distribution, hyperplasia and cytological atypia), intermediate trophoblast and marked cytologic atypia (Sebire et al., 2003a; Sebire et al., 2003b). These criteria are typically used for welldeveloped hydatidiform mole which gestational age at diagnosis is beyond 12 weeks (Sebire et al., 2003b; Merchant et al., 2005).

In this study, the significant histologic features that can distinguish CHM from PHM were central cistern, trophoblastic proliferation, trophoblastic atypia, two populations of villi, presence of fetal vessels and scalloped border. Howat et al. (1993) reported important features in differentiating CHM from PHM were atypical patterns of trophoblastic hyperplasia with circumferential and multifocal patterns. While Ishikawa (Ishikawa et al., 2009) found that the shape of villi and predominance of villi with hydropic change were useful to differentiate complete hydatidiform mole from partial hydatidiform mole. The insignificant pathological morphologies that distinguished CHM from PHM in this study were hydropic villi and trophoblastic inclusions which were similar to previous studies (Paradinas et al., 1996; Ishikawa et al., 2009). However, histological criteria for diagnosis type of hydatidiform mole are subjective and difficult to imitate. Furthermore, histological morphology overlaps between CHM and PHM, especially in early gestational age (Sebire et al., 2003a; Sebire et al., 2003b). All features can be detected in both subtypes of HM.

From previous studies, diagnosis of hydatidiform mole by histopathology had high intra- and inter-observer variation (Javey et al., 1979; Howat et al., 1993; Hoffner et al., 2010). In our study, the degree of agreement between two gynecologic pathologists for diagnosis was poor $(\mathrm{k}=0.373)$. Discordant diagnoses by H\&E between the two pathologists were $25.1 \%$, compared to $30-40 \%$ from previous literatures (Howat et al., 1993; Hoffner et al., 2010). More than half of PHM cases in this study had discordant diagnoses which were also comparable to a previous study (Garcia et al., 2008). This high rate of discordancy between experienced pathologists and indistinctive histologic criteria for diagnosis between CHM and PHM may make routine duties and services for pathologists more challenging. 
Nowadays, we can diagnose HM at an earlier gestational age, compared to previous decades (Mosher et al., 1998; Kim et al., 2004; Lertkhachonsuk et al., 2012a). As a result, this lesser gestational age leads to a more incomplete development of typical molar morphology such as diffuse trophoblastic hyperplasia and central villous cavitation (Genest et al., 2002; Merchant et al., 2005; Popiolek et al., 2006). In addition, criteria for diagnosis in early CHM overlap with PHM include minimal villous cavitation, mild villous hydrops, presence of fetal blood vessels, irregular villous, and villous pseudo-inclusion (Sebire et al., 2003b). In this study, 11 cases were histologically diagnosed as PHM but revealed negative results for P57KIP2. Interestingly, most of these cases (8/11 cases) were diagnosed and evacuated in the first trimester which might explain discordant immunohistochemical result in some of these cases.

P57KIP2, a cyclin-dependent kinase inhibitor, is an imprinted gene located at the chromosome locus 11p15.5. Previous studies found P57KIP2 related to several human malignancies (Pateras et al., 2009; Xu et al., 2012). P57KIP2 immunohistochemistry is one of the tools to distinguish CHM from non-CHM (Merchant et al., 2005; Garcia et al., 2008; Petts et al., 2014; Sasaki et al., 2015). This technique is a rapid, simple, and cost-effective method. Previous studies showed that the concordance between histological diagnosis and P57KIP2 was very good (kappa=0.89) (Crisp et al., 2003). In this study, final diagnosis by H\&E concluded that there were $97 \mathrm{CHM}$ cases and 30 cases of PHM. After performing P57KIP2, 1/97 cases of CHM had positive P57KIP2 IHC and 11/30 cases of PHM had negative P57KIP2 IHC. The discordant diagnosis between $\mathrm{H} \& \mathrm{E}$ and P57KIP2 was $9.4 \%$ which was comparable to $4.4-42 \%$ from other studies, (Crisp et al., 2003; Merchant et al., 2005; Popiolek et al., 2006). Comparing with P57KIP2, sensitivity and specificity of diagnosis by $\mathrm{H} \& \mathrm{E}$ for CHM in our study were $89.7 \%$ and $95.0 \%$, respectively and higher than results from Popiolek DA, et al (53.9\% and 59.2\%) (Popiolek et al., 2006).

In this study, we found 107 complete hydatidiform mole from 127 hydatidiform mole cases $(84.3 \%)$ which was higher than in other reports (Jeffers et al., 1993; Lurain, 2010; Seckl et al., 2010; Seckl et al., 2013). However, this incidence is similar to studies from Japan and Korea (Jun et al., 2003; Ishikawa et al., 2009). Generally, the incidence of hydatidiform mole, espeacially CHM, is higher in Asia than other regions of the world. P57KIP2 immunohistochemical is useful for differentiating CHM from non-CHM and expressed in both PHM and non-molar hydropic abortus. Other diagnostic tools such as ploidy analysis, short tandem repeat genotyping are also useful to differentiate PHM from non-molar hydropic abortus.

Eleven cases of PHM which were classified by H\&E yielded negative P57KIP2. Eight of these had discordant pathological diagnoses between two pathologists. Therefore, the histologic features of these cases might be ambiguous. Apart from indecisive criteria in diagnosis of PHM, Hoffner et al. (2010) had described that false negative results may occur possibly due to mosaic loss of chromosome 11 in unusual molar pregnancies. On the other hand, 1 case was diagnosed as CHM in H\&E but had diffusely strong P57KIP2 staining. This unusual characteristic could be explained by mosaic or chimeric conception or CHM with retained maternal chromosome 11(Hoffner et al., 2008; Lurain, 2010). Further ploidy study should be performed to respond to these cases. From our results, the discordance between P57KIP2 and H\&E occurred in both CHM and PHM. Thus, we recommend applying P57KIP2 in every case of suspected hydatidiform mole. Additional genomic study should be considered in non-CHM and unusual CHM cases to separate non-CHM into PHM and hydropic abortus; and explain atypical molar histology.

Postmolar GTN were identified in 35 cases from this study which were composed of 34 cases of P57KIP2 negative cases $(31.8 \%)$ and 1 case of P57KIP2 positive case $(5 \%)$. Although patients with both $\mathrm{CHM}$ and PHM should be monitored with serial hCG, accurate sub-classification of hydatidiform mole is important to determine the actual risk of GTN and to correct disease prognosis. Additionally, the incidence of postmolar GTN after PHM is lower than CHM. The hCG surveillance program for GTN following two types of HM can be different. Prolonged surveillance causes significant patient anxiety and improper cost-effectiveness (Sebire et al., 2007).

In conclusion, histopathology alone has a limitation in giving accurate type of hydatidiform mole. P57KIP2 immunohistochemistry is practical and should be utilized to distinguish CHM from non-CHM in every case of suspected hydatidiform mole. Additional genomic study may be required in P57KIP2 positive cases to separate PHM from hydropic abortus.

\section{Acknowledgements}

We would like to thank Mrs. Saranya Namto (Division of Gynecologic Pathology and Cytology) for providing paraffin-embedded specimens, Mr. Wasan Punyasang for his statistical assistance and Mr. Preecha Ruangvejvorachai for his laboratory technical assistance. This research was supported by Ratchadaphiseksompotch Fund and Grant for International Research Integration: research Pyramid, Ratchadaphiseksomphot Endowment Fund, Faculty of Medicine, Chulalongkorn University.

\section{References}

Crisp H, Burton JL, Stewart R, et al (2003). Refining the diagnosis of hydatidiform mole: image ploidy analysis and p57KIP2 immunohistochemistry. Histopathol, 43, 363-73.

Fukunaga M, Katabuchi H, Nagasaka T, et al (2005). Interobserver and intraobserver variability in the diagnosis of hydatidiform mole. Am J Surg Pathol, 29, 942-7.

Garcia-Barriola V, de Gomez MN, Dickson-Gonzalez S, et al (2008). Utility of p57 protein(KIP2) in molar disease to determine its androgenetic origin. J Reprod Med, 53, 476-80.

Genest DR, Dorfman DM, Castrillon DH (2002). Ploidy and imprinting in hydatidiform moles. Complementary use of flow cytometry and immunohistochemistry of the imprinted gene product p57KIP2 to assist molar classification. J Reprod Med, 47, 342-6. 


\section{Surang Triratanachat et al}

Hancock BW, Nazir K, Everard JE (2006). Persistent gestational trophoblastic neoplasia after partial hydatidiform mole incidence and outcome. J Reprod Med, 51, 764-6.

Hoffner L, Dunn J, Esposito N, et al (2008). P57KIP2 immunostaining and molecular cytogenetics: combined approach aids in diagnosis of morphologically challenging cases with molar phenotype and in detecting androgenetic cell lines in mosaic/chimeric conceptions. Hum Pathol, 39, 63-72.

Hoffner L, Parks WT, Swerdlow SH, et al (2010). Simultaneous detection of imprinted gene expression (p57(KIP2)) and molecular cytogenetics (FICTION) in the evaluation of molar pregnancies. J Reprod Med, 55, 219-28.

Howat AJ, Beck S, Fox H, et al (1993). Can histopathologists reliably diagnose molar pregnancy? J Clin Pathol, 46, 599-602.

Ishikawa N, Harada Y, Tokuyasu Y, et al (2009). Re-evaluation of the histological criteria for complete hydatidiform mole: Comparison with the immunohistochemical diagnosis using p57KIP2 and CD34. Biomed Res, 30, 141-7.

Javey H, Borazjani G, Behmard S, et al (1979). Discrepancies in the histological diagnosis of hydatidiform mole. Br J Obstet Gynaecol, 86, 480-3.

Jeffers MD, O’Dwyer P, Curran B, et al (1993). Partial hydatidiform mole: a common but underdiagnosed condition. A 3-year retrospective clinicopathological and DNA flow cytometric analysis. Int J Gynecol Pathol, 12, 315-23.

Jun SY, Ro JY, Kim KR (2003). p57kip2 is useful in the classification and differential diagnosis of complete and partial hydatidiform moles. Histopathol, 43, 17-25.

Kim SJ, Lee C, Kwon SY, et al (2004). Studying changes in the incidence, diagnosis and management of GTD: the South Korean model. J Reprod Med, 49, 643-54.

Lertkhachonsuk A, Israngura N, Tangtrakul S, et al (2012a). Complete hydatidiform mole change in clinical profile over three decades. J Reproductive Med, 57, 470-4.

Lertkhachonsuk R, Tantbirojn P, Paiwattananupant K (2012b). PTEN and MDM2 expression in the prediction of postmolar gestational trophoblastic neoplasia. J Reprod Med, 57, 333-40.

Lurain JR (2010). Gestational trophoblastic disease I: epidemiology, pathology, clinical presentation and diagnosis of gestational trophoblastic disease, and management of hydatidiform mole. Am J Obstet Gynecol, 203, 531-9.

Merchant SH, Amin MB, Viswanatha DS, et al (2005). p57KIP2 immunohistochemistry in early molar pregnancies: emphasis on its complementary role in the differential diagnosis of hydropic abortuses. Hum Pathol, 36, 180-6.

Mosher R, Goldstein DP, Berkowitz R, et al (1998). Complete hydatidiform mole. Comparison of clinicopathologic features, current and past. J Reprod Med, 43, 21-7.

Ngan HY, Kohorn EI, Cole LA, et al (2012). Trophoblastic disease. Int J Gynaecol Obstet, 119, 130-6.

Paradinas FJ, Browne P, Fisher RA, et al (1996). A clinical, histopathological and flow cytometric study of 149 complete moles, 146 partial moles and 107 non-molar hydropic abortions. Histopathol, 28, 101-10.

Pateras IS, Apostolopoulou K, Niforou K, et al (2009). p57KIP2: "Kip"ing the cell under control. Mol Cancer Res, 7, 1902-19.

Petts G, Fisher RA, Short D, et al (2014). Histopathological and immunohistochemical features of early hydatidiform mole in relation to subsequent development of persistent gestational trophoblastic disease. J Reprod Med, 59, 213-20.

Popiolek DA, Yee H, Mittal K, et al (2006). Multiplex short tandem repeat DNA analysis confirms the accuracy of p57(KIP2) immunostaining in the diagnosis of complete hydatidiform mole. Hum Pathol, 37, 1426-34.

Sasaki S, Sasaki Y, Kunimura T, et al (2015). Clinical Usefulness of Immunohistochemical Staining of p57 kip2 for the Differential Diagnosis of Complete Mole. Biomed Res Int, 2015, 905648.

Sebire NJ, Fisher RA, Rees HC (2003a). Histopathological diagnosis of partial and complete hydatidiform mole in the first trimester of pregnancy. Pediatr Dev Pathol, 6, 69-77.

Sebire NJ, Foskett M, Short D, et al (2007). Shortened duration of human chorionic gonadotrophin surveillance following complete or partial hydatidiform mole: evidence for revised protocol of a UK regional trophoblastic disease unit. BJOG, 114, 760-2.

Sebire NJ, Makrydimas G, Agnantis NJ, et al (2003b). Updated diagnostic criteria for partial and complete hydatidiform moles in early pregnancy. Anticancer Res, 23, 1723-8.

Seckl MJ, Sebire NJ, Berkowitz RS (2010). Gestational trophoblastic disease. Lancet, 376, 717-29.

Seckl MJ, Sebire NJ, Fisher RA, et al (2013). Gestational trophoblastic disease: ESMO Clinical Practice Guidelines for diagnosis, treatment and follow-up. Ann Oncol, 24, 39-50.

Wairachpanich V, Limpongsanurak S, Lertkhachonsuk R (2015). Epidemiology of hydatidiform moles in a tertiary hospital in Thailand over two decades: impact of the national health policy. Asian Pac J Cancer Prev, 16, 8321-5.

Xu XY, Wang WQ, Zhang L, et al (2012). Clinical implications of p57 KIP2 expression in breast cancer. Asian Pac J Cancer Prev, 13, 5033-6. 